\title{
A Group Tour Guide System with RFIDs and Wireless Sensor Networks
}

\author{
Po Yu Chen \\ Institute of Communications \\ Engineering \\ National Tsing Hua University \\ Hsin-Chu, 300, Taiwan
}

\author{
Wen Tseun Chen and \\ Cheng Han Wu \\ Department of Computer \\ Science \\ National Tsing Hua University \\ Hsin-Chu, 300, Taiwan
}

\author{
Yu-Chee Tseng and \\ Chi-Fu Huang \\ Department of Computer \\ Science \\ National Chiao Tung University \\ Hsin-Chu, 300, Taiwan
}

\begin{abstract}
This paper proposes a new application framework for group tour guiding services based on RFIDs and wireless sensor network. We consider a sensing field mixed with multiple independent tourist groups, each with a leader and several members. Members of a group will follow the moving path of their leader, but may occasionally roam around randomly based on their interest. Sensor nodes have to track leaders' locations and maintain following paths from members to leaders. A member may ask where his/her leader is, and a leader may "recall" his/her members. We propose a feasible solution to such an application by using existing technologies. A group guiding protocol is presented. The design enables reliable group guiding at low cost and low traffic load.
\end{abstract}

Categories and Subject Descriptors: C.2.1[Network Architecture and Design]: Wireless communication

General Terms: Algorithms, Design, Management

Keywords: RFID, tour guiding, wireless sensor network

\section{INTRODUCTION}

This work considers group guiding where tourists may form groups. The problem requirements are as follows: (1) Tourists in the same group may have similar behaviors, but with a certain degree of freedom. For example, they are likely to be in proximity but not necessarily always so. (2) The tour guide can broadcast instructions to members. On the contrary, a member may ask for information from the guide. (3) A member may get lost and need to locate the tour guide from time to time. (4) Multiple groups may coexist and their members may mix in the same physical environment. Although many navigation applications have been proposed for WSNs [2, 3, $4,5]$, the group guiding application has not been well addressed.

In this work, we look for feasible solutions to the group guiding problem by using RFIDs and WSNs. We propose a group guiding framework as follows. In the sensing field, a WSN is deployed for the purpose of location tracking by measuring audio signals. Each tourist group has one tour guide and some members. Only the tour guide carries a badge, which can emit audio signals for tracking purpose. Each member simply carries a ticket tagged with a passive RFID tag. Therefore, only the locations of tour guides can be tracked. Each node in the WSN is equipped with a "direction board", which is a LED panel that can show some basic information. Also, some sensor nodes are designated as "help centers", each of which is connected to a RFID reader and a laptop,

Copyright is held by the author/owner(s).

IPSN'07, April 25-27, 2007, Cambridge, Massachusetts, USA. ACM 978-1-59593-638-7/07/0004.

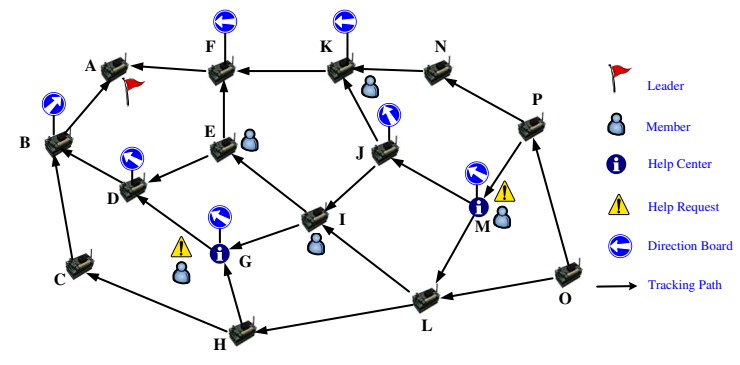

Figure 1: System architecture.

to provide more detailed guiding services. Our design goal is to reduce the management efforts. So most work will be done at the infrastructure side, and only the minimum amounts of devices need to be carried by users.

\section{SYSTEM ARCHITECTURE}

Consider a WSN deployed in a sensing field with one or multiple tourist groups. Each group has one leader and some members. Our goal is to provide the following services: (i) tracking the locations of leaders, (ii) maintaining the guiding paths to each leader, (iii) showing guiding paths for lost members, and (iv) helping leaders call their members. The system architecture is shown in Fig. 1. Each group leader carries a badge that can emit $4 \mathrm{kHz}$ audio signals to allow the WSN to track its location. Each group member simply carries a ticket with a passive RFID tag containing a group ID. Each sensor node is attached to a direction board for displaying simple guiding direction. Some nodes in the WSN are designated as help centers, each connected to a laptop and a RFID reader. A group guiding protocol is run at each sensor node. Below, we present three service scenarios in our system:

1. Leader tracking: At normal time, each badge will broadcast signals periodically. Sensor nodes cooperate to track the locations of group leaders and maintain the guiding path from each sensor node to each leader. Examples of tracking paths are shown in Fig. 1.

2. Help service: When a member gets lost, he/she can go to any help center and present his/her ticket to the RFID reader. Then guiding directions can be shown on the screen of the help center as well as the direction boards of those sensors which form a guiding path toward the sensor that is tracking the leader. Fig. 1 shows some help centers and direction boards.

3. Member-Recall: A group leader can also call his/her members back by pushing a button on the badge. A broadcast 


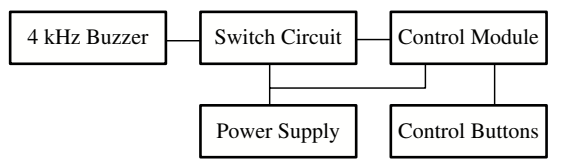

Figure 2: Modules of the leader badge.

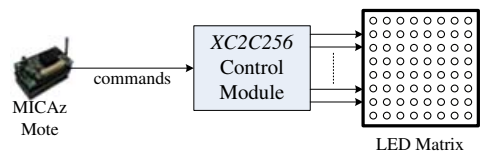

Figure 3: Direction board.

message will be flooded to the network. All direction boards on sensors will show the guiding directions to the sensor that is tracking the leader.

\subsection{Leaders' Badges}

Each badge will periodically broadcast audio signals for the WSN to track its location. We avoid using RF transmitters to keep the cost low. A badge is composed of a buzzer, a switch circuit, a control module, some control buttons, and a power supply, as shown in Fig. 2. The buzzer can transmit $4 \mathrm{kHz}$ sound in a certain pattern specified by the control module. The switch circuit passes control messages from the control module to the buzzer. The control module is implemented by a Xilinx XC2C256 chip.

\subsection{Sensor Nodes}

The sensor nodes are realized by MICAz motes, which can sense sounds through their microphones. Each sensor node is connected to a direction board, which is an $8 \times 8$ LED matrix, as shown in Fig. 3. Implemented by a Xilinx XC2C256 chip, the control module is connected to the sensor node via a 51-pin connector. The LED matrix can show in red for lost members or in green for recalling members. Direction boards are to support "on-site" visualization. Our current implementation simply displays group IDs and guiding directions interchangeably.

\subsection{Help Centers}

A help center is a laptop connected to a sensor node and a RFID reader, as shown in Fig. 4. We adopt the MPR1230 RFID reader by AWID Inc. [1]. The mappings between tags and group IDs are stored in a Microsoft SQL server. Each help center can provide the following services. First, given a group ID, it will find the location of the group leader and ask sensors on the path toward the leader to display instructions on their direction boards. Second, the navigation paths will also be shown on its screen so that the user can have a global view about leader location. Traditional help centers are usually located in limited areas such as entrances. Enabled by pervasive sensing and communication technologies, our system can virtually deploy help centers everywhere.

\section{IMPLEMENTATION}

We have developed a prototype of the proposed system. Fig. 5 shows all devices developed in a small-scale environment with 16 $M I C A z$ motes, two help centers and two leader badges. The network control module and our group guiding protocol are embedded in sensor nodes. The group guiding protocol can be verified by checking the LEDs on sensor nodes. The DIP switches on the leader badge are used to switch badge's states. We design two user interfaces. Fig. 6(a) shows the administrative interface for managing the mapping between group ID and tag ID to be used in the ticket booth. Fig. 6(b) is the interface to be used by members at the help centers. The left lower part contains the group information

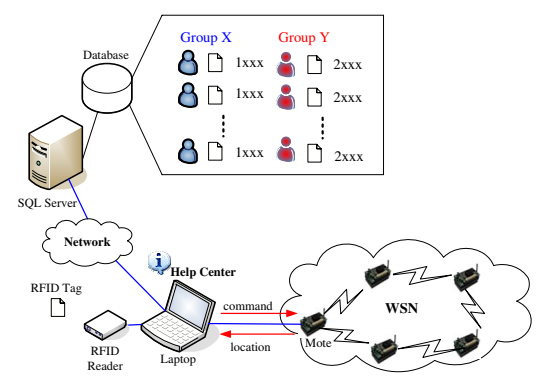

Figure 4: Help center.

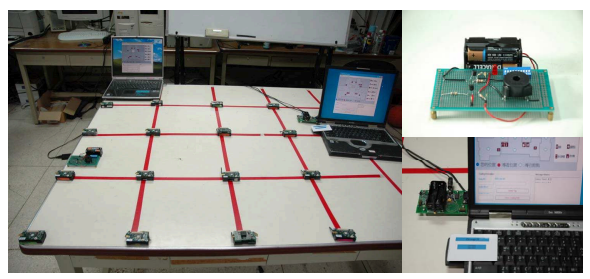

Figure 5: Environment overview.

and the upper part shows the navigation information. The framework places special emphasis on the feasibility of the model, so what each user needs to carry is only a ticket. This could minimize the management efforts. Although our current prototyping only contains very brief guiding information, we believe that it can trigger more services with richer contents.

\section{ACKNOWLEDGMENTS}

This work is co-sponsored by Taiwan MoE ATU Program, by NSC grants 93-2752-E-007-001-PAE, 96-2623-7-009-002-ET, 952219-E-009-007, 95-2218-E-009-209, and 94-2219-E-007-009, by MOEA under grant number 94-EC-17-A-04-S1-044, by ITRI, Taiwan, and by Intel Inc.

\section{REFERENCES}

[1] AWID Inc. http://www.awid.com.

[2] C. Buragohain, D. Agrawal, and S. Suri. Distributed navigation algorithms for sensor networks. In IEEE INFOCOM, 2006.

[3] P. Corke, R. Peterson, and D. Rus. Localization and navigation assisted by cooperating networked sensors and robots. Int. Journal of Robotics Research, 24(9):771-786, Oct. 2005.

[4] Q. Li, M. Rosa, and D. Rus. Distributed algorithms for guiding navigation across a sensor network. In ACM Int'l Conf. on Mobile Computing and Networking (MobiCom), pages 313-325, 2003.

[5] Y.-C. Tseng, M.-S. Pan, and Y.-Y. Tsai. A Distributed Emergency Navigation Algorithm for Wireless Sensor Networks. IEEE Computers, 39(7):55-62, July 2006.

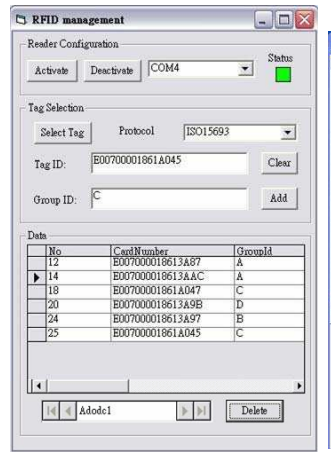

(a)

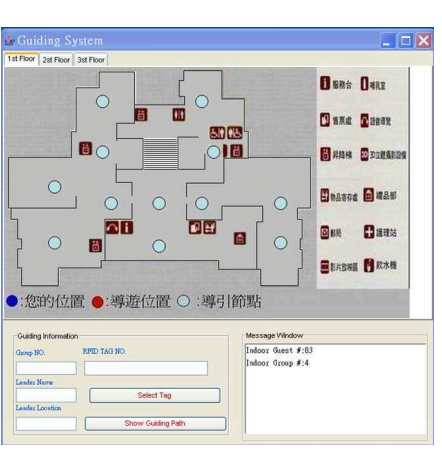

(b)
Figure 6: GUI of the help center (a) administrator and (b) guests. 